\title{
Aprendizaje basado en problemas y motivación en estudiantes de educación física
}

\section{Motivation-based learning in physical education students}

\author{
Damián Fernando Montalván-Larco \\ dmontalvanl@ucacue.edu.ec \\ Universidad Católica de Cuenca, Azogues - Ecuador \\ https://orcid.org/0000-0001-6951-8041 \\ Darwin Gabriel García-Herrera \\ dggarciah@ucacue.edu.ec \\ Universidad Católica de Cuenca, Azogues - Ecuador \\ https://orcid.org/0000-0001-6813-8100 \\ Carlos Marcelo Ávila-Mediavilla \\ cavilam@ucacue.edu.ec \\ Universidad Católica de Cuenca, Cuenca - Ecuador \\ https://orcid.org/0000-0002-2649-9634 \\ Juan Carlos Erazo-Álvarez \\ jcerazo@ucacue.edu.ec \\ Universidad Católica de Cuenca, Cuenca - Ecuador \\ https://orcid.org/0000-0001-6480-2270
}

Recepción: 11 abril 2020

Revisado: 16 de mayo 2020

Aprobación: 15 junio 2020

Publicación: 1 de julio 2020 


\title{
RESUMEN
}

El objetivo de la investigación fue analizar la relación del aprendizaje basado en problemas en la motivación de los estudiantes en el área de la educación física. De tipo descriptivo correlacional no experimental transeccional. La prueba Chi-cuadrado de Pearson determina que existe relación directa entre las variables de estudio, aunque al no existir significancia bilateral, se destaca que la motivación podría verse influenciada por factores externos al ABP. Se concluye que estudiantes tienen consideraciones favorables para el $A B P$, puesto que mencionan haber aprendido con este método, percibiéndose motivados al aprendizaje cooperativo. El ABP permite a los estudiantes al auto descubrimiento de sus capacidades, habilidades, fortalezas, conduciendo a la generación de un aprendizaje centrado en la indagación, reflexión, como medio para comprender la importancia del auto aprendizaje como estrategia no solo para aprender sobre un momento académico especifico, sino, para valorar el aprender permanente a lo largo de la vida.

Descriptores: Aprendizaje activo; autoaprendizaje; educación física; motivación. (Palabras tomadas del Tesauro UNESCO).

\begin{abstract}
The objective of the research was to analyze the relationship of problem-based learning to student motivation in the area of physical education. Descriptive, correlational, nonexperimental, transectional. Pearson's Chi-square test determines that there is a direct relationship between the study variables, although since there was no bilateral significance, it is highlighted that motivation could be influenced by factors external to PBL. It is concluded that students have favorable considerations for the ABP, since they mention having learned with this method, perceiving themselves motivated to cooperative learning. The ABP allows students to self-discover their capacities, abilities, strengths, leading to the generation of learning focused on inquiry, reflection, as a means to understand the importance of self-learning as a strategy not only to learn about an academic moment. I specify, if not, to value lifelong learning throughout life.
\end{abstract}

Descriptor: Activity learning; self instruction; physical education; motivation. (Words taken from the UNESCO Thesaurus). 


\section{INTRODUCCIÓN}

El constante cambio en la sociedad fruto de descubrimientos y avances tecnológicos conllevan a que la educación, las metodologías educativas, los procesos y recursos formativos también den un giro muy importante, aquella persona que se mantenga en solo un sistema de educación tradicional está condenado al fracaso o en su defecto a escasas oportunidades de progreso. Los nuevos modelos educativos se acoplan a las diversas materias o Áreas de estudio y cada vez más a las realidades sociales de nuestra educación que requieren de un alto grado de responsabilidad y de la adquisición de conocimientos (Tapia-Vélez, et al., 2020).

Existen un gran número de investigaciones realizadas en las metodologías activas, Aprendizaje Basado en Problemas, en varios países se han dado muy buenos resultados y han sido aplicadas en varias áreas de forma acertada y estas en su gran mayoría enfocados en el campo de la educación han logrado generar óptimos resultados, para ello hacemos un aporte extra para que las metodologías activas (ABP) sean aplicadas en la motivación de los estudiantes para mejorar su aprendizaje en el área de la Educación Física.

En este sentido, (Byrne, 2009) en sus estudios realizados en EEUU sobre la motivación en la educación física, resaltan que los estudiantes mantienen una actitud buena en el momento de realizar trabajos con acompañamiento por parte del docente, que realiza un papel de guía en este proceso, además mantienen que una pedagogía tradicional no es muy aceptable por los estudiantes disminuye su aspecto de la motivación mientras que la motivación con efecto pedagógico actual, nuevo, activo varia totalmente su motivación siendo está muy buena para los aprendizajes de los estudiantes.

Para partir de cómo mejorar la educación física (Méndez-Alonso, Méndez-Giménez, \& Fernández-Río, 2016) interpretaron, que para llegar a tener docentes de calidad es necesario que desde su formación como profesional sepan manejar, estrategias, métodos, modelos pedagógicos actuales, se debe al docente actual enseñarle más en que enseñar. Y tiene mucha razón ya que hay docentes que vienen con una formación 
de cómo sus docentes enseñaron ya que desconocen el uso de estrategias activas actuales y solo se centraron en enseñarles de forma tradicional.

Para (Cheaney, 2005) menciona que al aplicar el ABP por primera vez en los tiempos modernos, es muy importante cada vez en la reforma educativa de todo el mundo puesto que expone al estúdiate a problemas del mundo real en un contexto que fomenta al estudiante el trabajar en grupos, la cal sirve para que su aprendizaje sea mediante la investigación y la conversación a resolver los problemas planteados, por cuanto se vuelve difícil enseñar algunos temas de manera de conferencia, en sus estudios aplicó el ABP, en varias materias y ha tenido buenos resultados de aprendizajes.

Es por ello que (Morales, 2004) en su investigación identifican que una enseñanza tradicional muy difícilmente puede contribuir a desarrollar ciertas habilidades y capacidades de competencia en los estudiantes, es por eso que la educación y los procesos de enseñanza necesitan un cabio con estrategias activas nuevas y usan al ABP como una de ellas, para que signifique y haga a la clase mucho más interesante, participativa, protagonista del aprendizaje al estudiante y que este a su vez desarrolle habilidades, capacidades y actitudes para su entorno de estudio.

EL uso del ABP es una estrategia que debemos saber y entender, que es muy positiva para los procesos de aprendizaje, por su forma de ser y ayudar a los estudiantes a comprender una mejor manera los problemas que se presenten en la materia, es una metodología totalmente distinta a una tradicional. Se podría también decir que, en el ABP, se encuentra una herramienta fabulosa para la enseñanza donde el docente centra al estudiante como protagonista de su propio aprendizaje. Además, el estudiante crea una actitud diferente trabajando en equipo, ayudando al resto de compañeros a tener una mayor motivación para que juntos puedan alcanzar el éxito en sus conocimientos (Romero-Ramírez, 2020).

$E L$ uso del $A B P$, es una estrategia que debemos saber y entender, que es muy positiva en nuestras clases por su forma de ser y ayudar a los estudiantes a comprender una mejor manera los problemas que se presenten en la materia, es una metodología 
totalmente distinta a una tradicional. Se podría también decir que, en el $A B P$, se encuentra una herramienta fabulosa para la enseñanza donde el docente centra al estudiante como el eje principal de su aprendizaje. Además, el estudiante crea una actitud diferente trabajando en equipo, ayudando al resto de compañeros a tener una mayor motivación para que juntos puedan alcanzar el éxito en sus conocimientos.

Para (Remacha Irure \& Belletich, 2015) quieren demostrar que el estudiante aprende a resolver problemas a partir dela búsqueda de información y con el uso del razonamiento científico con el uso del ABP, pero esta vez lo hizo con ABP por proyectos lo cual el resultado fue totalmente favorecedor y lo hizo con la finalidad de que el docente se convierte en un transmisor de conocimientos nuevos, con motivación en sus clases. Pero el ABP por problemas igualmente es muy favorecedor que se plantean para darle soluciones juntos ya que el docente siempre debe guiar a los estudiantes. El docente debe saber y reconocer que el ABP, tiene como esencia el describir, identificar y analizar los problemas para poder resolver y esto tiene un gran éxito cuando la interacción se d entre docente y alumno.

En su estudio realizado (Paredes-Curin, 2016) resalta que los estudiantes muestran nociones he indica que las habilidades que el ABP, permiten desarrollar o a su vez esta les ayudo fortalecer durante su estudio y el desarrollo de una problemática. Él nos menciona que el $\mathrm{ABP}$, tiene 3 habilidades que son. Habilidad de identificar el problema, habilidad de desarrollar el problema, habilidad de exponer el trabajo

De la misma manera (Zapatero, 2017) menciona que las metodologías activas deber ir centradas en los alumnos para mejorar su educación y que estas puedan sostener un buen uso de metodologías en el aula, en varios de sus estudios los resultados le demuestran que la mayoría de docentes siguen un estilo tradicional que solo gira en torno al docente, es por ello que plantea el uso de estrategias y metodologías nuevas (ABP) para alcanzar niveles de motivación más óptimos en los estudiantes y el alcance satisfactorio en su trabajo. 
Por otro lado, (Rodríguez M., 2014) manifiesta que las metodologías usadas como el $\mathrm{ABP}$, en su campo de estudios ha tenido buenos resultados, más aún la falta de conocimientos en el área de la medicina y podemos darnos cuenta que esta metodología es aplicable para todos sigue siendo este un principal problema en la educación y formación del estudiante. Por ello los docentes podemos darnos cuenta que esta metodología es adaptable para cualquier área de estudio, y aplicable para sus procesos de aprendizaje.

\section{EI ABP: una metodología activa}

En este sentido, (Luy-Montejo, 2019) define al ABP como un tipo de metodología activa, de enseñanza que va dirigida específicamente al estudiante y lo menciona como el eje central en este proceso, además menciona que esta metodología se encarga por producir un aprendizaje en el estudiante de solucionar problemas, con sus compañeros y docente, en este estudio con el ABP se lo llevo a un sentido de estudio más producto en el impacto de su inteligencia emocional y los resultados de su aprendizaje fueron excelentes.

Cabe decir que (Castaño, 2015) distingue al ABP como la mejora estrategia, por el intento de resolver problemas del mundo real sin muchas veces tener una base teórica, es decir que el aprendizaje y habilidades se desarrollan en proceso que van resolviendo los problemas, por lo general el tiempo invertido para resolver el problema es largo y es aquí donde el docente debe mantener a estudiante motivado durante este proceso y poder ir de las materias más básicas a las más complejas siendo esta metodología apta para Matemáticas, EEFF, Lengua, etc., y en todas podrá ser utilizada de una manera muy eficaz.

\section{La educación física y su influencia en la educación.}

Es por eso que (Betancur, 2018) en sus estudios menciona que a la educación física se le debe dar una mirada diferente en la formación física y motriz del hombre, para que este a su vez pueda centrarse, diseñar, emplear un trabajo diferente en el que el estudiante 
sepa manejar los tipos de metodologías o estrategias que le enseña su profesor, desde los inicios de sus prácticas en la docencia ellos usan estrategias metodológicas y que estas dan un gran efecto en su momento de trabajar y estas puedan enriquecer a su formación y desarrollo de sus conocimientos en el aula.

Varios investigadores le dan a la educación física un papel muy importante en la educción, pero (Ventura, 2018) en sus estudios definen que la educación física mejora y ayuda en la desinhibición del estudiante que tiene en el aula y esto se da atreves del juego y la práctica pre deportiva que otorga la educación física ya que ayuda a su relación social y a expresarse de una forma natural para mejorar su vínculo con la sociedad.

\section{La motivación en la educación.}

La motivación juega un papel importante en la educación por ello es que (Ospina Rodríguez, 2006) indica que la motivación da un aspecto relevante en el proceso de aprendizaje ya que tiene un sentido de doble vía que se debe tener, la razón es que involucra al estudiante y docente que entran en el juego para mejorar el aprendizaje, además hace hincapié que el docente debe favorecer a la motivación intrínseca ya que es muy importante para los procesos desmotivación en los aprendizajes.

Los docentes deberíamos cuestionar si en verdad hay o no motivación en nuestras clases como lo manifiesta (García de las Bayonas Plazas \& Baena - Extremera, 2017) cuestionan al docente en su forma de transmitir conocimientos y su motivación para este proceso, ya que hay que considerar al estudiante como un agente activo al que hay que motivar y presentar nuestras clases de una manera diferente desde una tarea, deber 0 una proyección pero que sea de una manera innovadora, diferente con imágenes acorde a su edad serie caricaturas que él se sienta a gusto y feliz de estar en esa clase que sea diferente al resto, de esta manera se va a ver una mejora en la motivación del estudiante y nuestro papel como presentador de los temas sea más dinámico. 


\section{Efectos de la motivación.}

En este sentido, (Salgado, et al., 2017) identifica que existen varios tipos de personas para los diversos cambios de actitud en la motivación de los aprendizajes, pero a cada uno de ellos les ayuda a mejorar sus aprendizajes la parte de influencia del docente, con su interacción dinámica en grupo y la determinación de la motivación en sus trabajos. Por ende, podemos decir que es de suma importancia que el guía o profesor conozca y maneje estrategias metodológicas nuevas, dinámicas en grupo, trabajos motivacionales, charlas que impulsen a sus estudiantes a querer aprender más por cuanto con esas actividades sus clases y la comunicación con sus estudiantes mejorara notablemente la motivación en ellos y se podrán notar cabios muy buenos en cada uno.

En función de lo planteado, se ha observado la Ciudad de Cuenca no se ha realizado un estudio basado en la motivación que influya en la Educación Física al momento de trabajar con el ABP, siendo evidenciado por (Palta \& Siguencia, 2018), quienes realizaron una investigación del ABP, en el cantón Paute, provincia del Azuay, la cual manifiestan que se identificó el nivel de conocimientos y la utilización actual de esta estrategia didáctica, realizando un análisis profundo en sus planificaciones, identificando en esta investigación que el conocimiento del $A B P$, es casi nulo tanto en docentes como en estudiantes.

Para ello el objetivo de la investigación es analizar la relación del aprendizaje basado en problemas en la motivación de los estudiantes en el área de la educación física del cantón Paute, provincia del Azuay-Ecuador.

\section{MÉTODO}

La investigación presenta un carácter descriptivo correlacional no experimental transeccional, recopilándose información en un único momento con la finalidad de conocer la relación de las variables de estudio, mediante análisis de Chi-cuadrado de Pearson (Hernández, Fernández, \& Baptista, 2010). El muestreo utilizado fue de tipo aleatorio estratificado en 62 estudiantes de la asignatura de educación física del cantón 
Paute, provincia del Azuay-Ecuador. Para la recolección de datos, se usó la técnica de la encuesta, a través de un cuestionario con valoración de escala de Likert, aplicado online mediante formulario de Google. Es importante además indicar que el cuestionario fue validado mediante coeficiente de Alfa de Cronbach; obteniéndose un resultado de 0,92 catalogándose confiable para su aplicación, lo datos se analizaron estadísticamente mediante el programa SPSS V23 (Rodríguez, Erazo \& Narváez, 2019).

\section{RESULTADOS}

\section{Tabla 1}

Su motivación mejoró al trabajar con sus compañeros con el ABP.

\begin{tabular}{llcccc} 
& & Frecuencia & Porcentaje & $\begin{array}{c}\text { Porcentaje } \\
\text { válido }\end{array}$ & Porcentaje acumulado \\
\hline Válido & Neutral & 1 & 1,4 & 1,6 & 1,6 \\
& Muy satisfecho & 61 & 85,9 & 98,4 & 100,0 \\
& & & & & \\
& Total & 62 & 87,3 & 100,0 & \\
Total & Sistema & 9 & 12,7 & & \\
\hline
\end{tabular}

Fuente: Elaboración propia

La tabla 1 expone los datos obtenidos de la indagación sobre una de las variables con mayor transcendencia en este estudio investigativo. La motivación de los estudiantes mejoró al trabajar con sus compañeros con el ABP. Los datos que arroja en el análisis es de un $98,4 \%$ de los encuestados consideran que el trabajar con ABP en la educación física mejora su motivación y la de sus compañeros. Con esta afirmación el docente deja un precedente de que el trabajar con metodologías activas cambia de manera muy notable el interés y la motivación por parte de los estudiantes. 


\section{Tabla 2}

Su motivación mejoró al trabajar con sus compañeros con el ABP. * El docente manejo el desarrollo del tema*. Tabulación cruzada

\begin{tabular}{llrrr} 
& \multicolumn{2}{c}{ Manejo el desarrollo del tema. } & \\
& Neutral & Muy satisfecho & Total \\
\hline Su motivación mejoró & Neutral & 0 & 1 & 1 \\
& Muy satisfecho & 6 & 55 & 61 \\
Total & & 6 & 56 & 62
\end{tabular}

\section{Pruebas de chi-cuadrado}

$\begin{array}{cccccc} & \text { Valor } & \text { gl } & \begin{array}{c}\text { Sig. asintótica }(2 \\ \text { caras })\end{array} & \begin{array}{c}\text { Significación } \\ \text { exacta (2 caras) }\end{array} & \begin{array}{c}\text { Significación } \\ \text { exacta (1 cara) }\end{array} \\ \text { Chi-cuadrado de Pearson } & , 109^{a} & 1 & , 741 & & \\ \text { Corrección de continuidad } & \text { b } & , 000 & 1 & 1,000 & \\ \quad \text { Razón de verosimilitud } & , 205 & 1 & , 650 & 1,000 & , 903 \\ \text { Prueba exacta de Fisher } & , 107 & 1 & , 743 & & \\ \text { Asociación lineal por lineal } & 62 & & & & \\ \text { N de casos válidos } & 62 & & \end{array}$

a. 2 casillas $(50,0 \%)$ han esperado un recuento menor que 5 . El recuento mínimo esperado es, 10 .

b. Sólo se ha calculado para una tabla $2 \times 2$

Fuente: Elaboración propia.

La prueba Chi-cuadrado de Pearson determina que existe relación directa entre las variables de estudio, aunque al no existir significancia bilateral, se destaca que la motivación podría verse influenciada por factores externos al ABP, destacándose la importancia de proseguir con esta metodología con la finalidad de incentivar cambios actitudinales de los estudiantes al logro de metas comunes. 


\section{Tabla 3}

Mejoró su compañerismo el trabajar con el ABP.

\begin{tabular}{llcccc} 
& & Frecuencia & Porcentaje & Porcentaje válido & $\begin{array}{c}\text { Porcentaje } \\
\text { acumulado }\end{array}$ \\
\hline Válido & Neutral & 5 & 7,0 & 8,2 & 8,2 \\
& Muy satisfecho & 56 & 78,9 & 91,8 & 100,0 \\
& Total & 61 & 85,9 & 100,0 & \\
Perdidos & Sistema & 10 & 14,1 & & \\
Total & & 71 & 100,0 & & \\
\hline
\end{tabular}

Fuente: Elaboración propia

En función de los resultados obtenidos, se evidencia que el uso de estrategias de aprendizaje basado en problemas puede llegar a cambiar de forma positiva las aptitudes de los estudiantes hacia el aprendizaje, vinculándose a la cooperación para alcanzar metas comunes, mejorando la visión de trabajo en equipo, lo cual trae consigo la praxis de valores sociales en los ambientes de clases.

\section{PROPUESTA}

La educación es la arma más fuerte que el ser humano tiene para poder luchar por sus ideales, es por esa razón que en los hombros del docente recae una gran responsabilidad en los procesos de enseñanza, más allá de lo que el enseña o que enseña es la manera de cómo, esta última parte es fundamental ya que hay o hacemos estudiantes que muchas de la veces los aprendizajes son momentáneos mas no significativos y esto se debe a la forma o manera en la que el docente enseña, se considera que un docente debe tener un estilo propio de enseñanza siempre y cuando este sea beneficioso y alimentarle para el estudiante es decir que los procesos de aprendizaje sean permanentes y estos en su vida a futuro sean aplicables, que le sirvan en su vida cotidiana es por ello que en mi investigación me dio buenos resultados y al estudiante le sirvió y consideró que en un futuro le podrá ayudar. 
Es por ello que como docente propongo que en el área de la Educación Física se use de manera más continua metodologías activas las que el docente quiera, pero antes de usarlas sepa manejarlas, las conozca bien caso contrario no va a servir en sus procesos de enseñanza. Varias Áreas de educación usan metodologías activas nuevas, diferentes las cumplan a su salón de clases y de verdad que son un éxito, es por ello que me atrevo y lo digo que el $A B P$, en la educación física tuvo un gran impacto en la motivación y procesos de aprendizajes en los estudiantes siendo está muy satisfactoria tanto para mí como docente y para los estudiantes como el eje central del desarrollo del aprendizaje, su trabajo de manera individual y grupal es muy bueno, la interacción entre ellos y la familiarización con el docente es fantástica, es por ello que el realizar actividades con metodologías activas va a darle a la clase un enfoque positivo y diferente que el estudiante se va a sentirse cómodo y muy a gusto. Para esto se debe tener en cuenta el siguiente proceso.

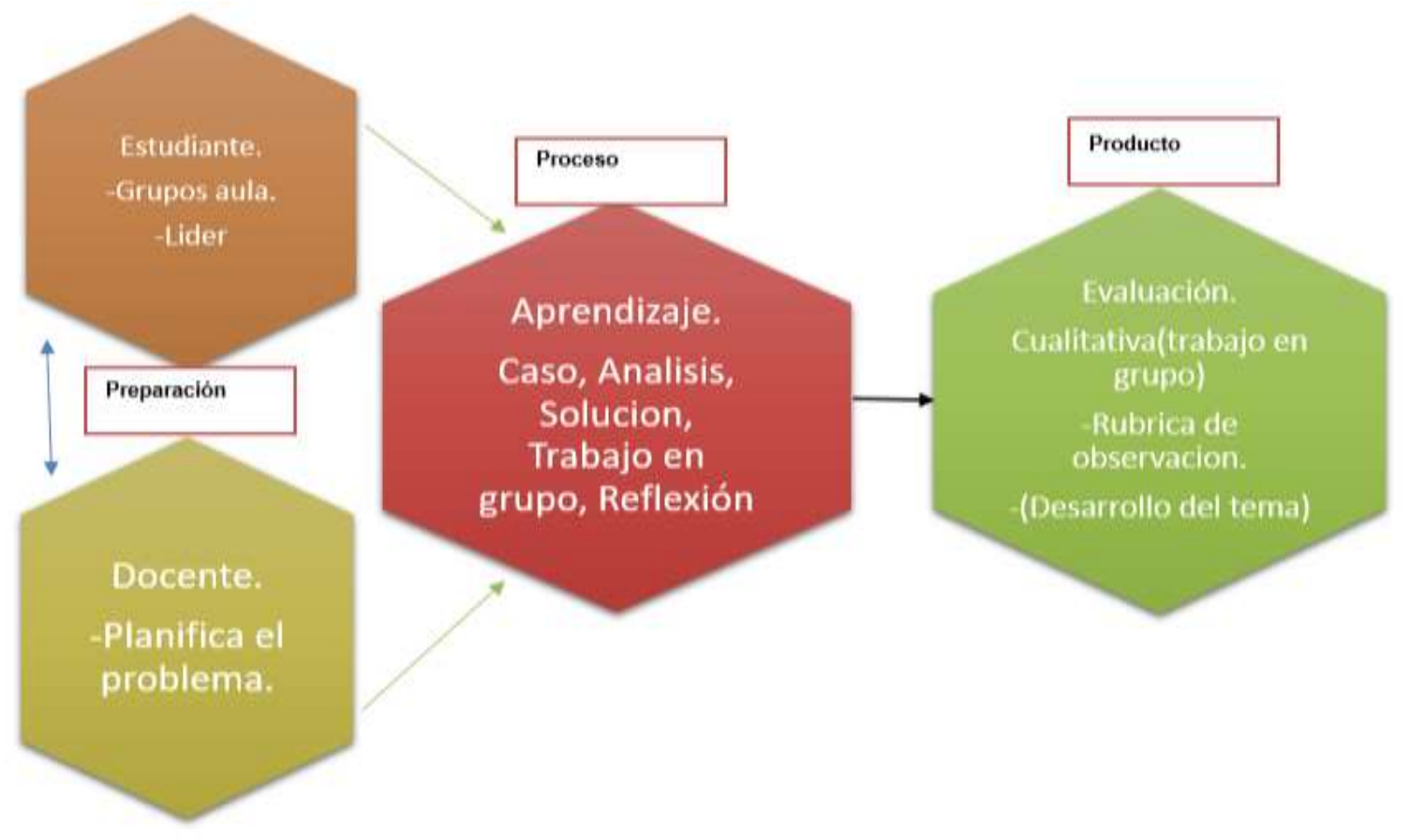

Figura 1. ABP basado en las 3P. Fuente: Elaboración propia. 
A continuación, se detalla la aplicación del ABP a través de las 3P

1. En la preparación el docente prepara la actividad la planifica, las actividades las plantea frente a los objetivos de aprendizaje para la sección de clase, selecciona la estrategia metodológica que va a utilizar, dialoga con los grupos sobre la preparación de materia, problema que se va impartir en los grupos, guía los procesos que se dan en la investigación asesora a cada uno miembros líderes de los grupos a trabajar, en el proceso cuida de los tiempos en los que se debe trabajar cada situación dúrate la discusión que se da en los grupos, en su producto evalúa cada una de las intervenciones y soluciones que le van dando al problema con los conocimientos y destrezas adquiridas en el proceso.

2. Investiga conceptos, ideas recopila información que le sea útil para dar solución al problema, analiza las ideas y situaciones que se van dando, sus materiales los va preparando acorde a el desarrollo de las ideas que tienen en su grupo prepara la presentación oral, y va seleccionando dinámicas de discusión he interacción en el grupo, en su proceso presenta al mismo las ideas más sustentables y aplicables a su desarrollo del tema, propone dinámicas para el desarrollo del caso y cómo van a ser expuestas por cada miembro asignándole una tarea o punto concreto que debe defender y dar su solución, en su producto reflexionan sobre lo que aprendieron y la repercusión en su desarrollo responden a las evaluaciones del docente e inquietudes de sus demás compañeros en la cual defiende su tema y solución al problema de manera conjunta con su grupo.

3. El docente analiza cada uno de los puntos a favor en los que el grupo de estudiantes se desenvolvieron y cada una de las soluciones que le dieron a su problema, analiza la participación de los estudiantes durante su exposición y el desenvolvimiento que tuvo el grupo, su producto en la evaluación cualitativa debe ser acorde a una rúbrica o lista de cotejo en la cual respalde su análisis y calificación que se asigne al grupo y sepa corroborar su nota con su evaluación. 


\section{CONCLUSIÓN}

Se concluye que estudiantes tienen consideraciones favorables para el ABP, puesto que mencionan haber aprendido con este método, percibiéndose motivados al aprendizaje cooperativo. El ABP permite a los estudiantes al auto descubrimiento de sus capacidades, habilidades, fortalezas, conduciendo a la generación de un aprendizaje centrado en la indagación, reflexión, como medio para comprender la importancia del auto aprendizaje como estrategia no solo para aprender sobre un momento académico especifico, sino, para valorar el aprender permanente a lo largo de la vida. La motivación se ve influenciada positivamente por el ABP, aunque no es el único factor que la incentiva en los estudiantes objeto de estudio.

\section{FINANCIAMIENTO}

No monetario

\section{AGRADECIMIENTOS}

A los estudiantes de educación física del cantón Paute, provincia del Azuay-Ecuador.

\section{REFERENCIAS}

Betancur, J. L. (2018). El docente de educación física y sus prácticas pedagógicas. [The physical education teacher and his pedagogical practices]. Revista Latinoamericana de Estudios Educativos, 1-15. doi: https://doi.org/10.17151/rlee.2018.14.1.2

Byrne, M. S. (2009). La Influencia de la educación deportiva en la motivacion del alumno en la educacion fisica. [The influence of sports education on student motivation in physical education]. Educacion Fisica y pedagogia deportiva, 253-206. doi:https://doi.org/10.1080/17408980801995239

Castaño, V. y. (2015). El método del aprendizaje basado en problemas como una herramienta para la enseñanza de las matemáticas. [The method of problembased learning as a tool for teaching mathematics]. Revista Iberoamericana para la Investigación y el Desarrollo Educativ, 1-13. Obtenido de https://n9.cl/aqj8 
Cheaney, J. D. (2005). Problem-based learning in an on-line. [Aprendizje basado en problemas en linea de curso biotecnologia]. Retrospective Theses and Dissertations, 11-34. Obtenido de https://n9.cl/m2gq

García de las Bayonas Plazas, M., \& Baena - Extremera, A. (2017). Educación física a través de diferentes metodologías didácticas. [Motivation in phisical education through different didactics methodologies]. Revista de Currículum y Formación de Profesorado, 387-402. Obtenido de https://n9.cl/rm5us

García-Cedeño, G., Vélez-Loor, M., Franco-Zambrano, C., \& Ormaza-Bermello, M. (2020). Educación por competencias: Un reordenamiento curricular durante emergencia escolar por COVID-19. [Competency Education: A Curricular Rearrangement During School Emergency by COVID-19]. EPISTEME KOINONIA, 3(5), 221-235. http://dx.doi.org/10.35381/e.k.v3i5.770

Luy-Montejo, C. (2019). El Aprendizaje Basado en Problemas (ABP) en el desarrollo de la inteligencia emocional de estudiantes. [Problem Based Learning (PBL) in the Development of Emotional]. Revista de Psicologia Educativa, 353 - 383. doi: http://dx.doi.org/10.20511/pyr2019.v7n2.288

Méndez-Alonso, D., Méndez-Giménez, A., \& Fernández-Río, J. (2016). Incorporación de las competencias básicas a la educación física en educación primaria [Integrating basic competences into physical education (pe) in primary education]. Revista Internacional de Medicina y Ciencias de la Actividad Física y del Deporte, 457-473. doi:http://dx.doi.org/10.15366/rimcafd2016.63.004

Morales, P. y. (2004). El Aprendizaje Basado en Problemas (ABP) en el desarrollo de la inteligencia emocional de estudiantes universitarios. [Problem Based Learning (PBL) in the Development of Emotional Intelligence of University Students]. Revista Theoria , 353 - 383. doi: http://dx.doi.org/10.20511/pyr2019.v7n2.288

Ospina Rodríguez, J. (2006). La motivación, motor del aprendizaje. [Motivation, the Engine of Learning]. Revista Ciencias de la Salud, 158-160. Obtenido de https://n9.cl/9r25

Palta, N., \& Siguencia, J. (2018). El Aprendizaje Basado en Problemas como estrategia didáctica en el proceso de enseñanza [Problem Based Learning as a didactic strategy in the teachingprocess]. Killkana Sociales., Vol. 2 (No. 2), 1-8. https://doi.org/10.26871/killkana_social.v2i2.291 
Paredes-Curin, C. R. (2016). Aprendizaje basado en problemas (ABP): Una estrategia de enseñanza de la educación $n$ estudiantes de un liceo municipal de Cañete [Problem-based Learning (PBL):A Teaching Strategy of Environmental Education, in Cañete Municipal School Students]. Revista Electrónica Educare, vol. 20(1), 126. doi: http://dx.doi.org/10.15359/ree.20-1.6

Remacha Irure, A., \& Belletich, O. (2015). El método de aprendizaje basado en proyectos (ABP)en contextos educativos rurales y socialmente desfavorecidos de la educación infantil [The project-based learning method (ABP) in rural and socially disadvantaged educational contexts of early childhood e]. Perspectiva Educacional, Formación de, vol. 54(núm. 1), 90-109. doi:DOI: 10.4151/07189729Vol.54-Iss.1-Art.294

Rodríguez M., S. L. (2014). El aprendizaje basado en problemas para la educación médica [Problem-based learning for medical education]. Revista Med., 32-36. Obtenido de https://n9.cl/2bbw

Romero-Ramírez, M. (2020). Vocación docente como respuesta esperanzadora en contextos de vínculos humanos frágiles. [Teaching vocation as a hopeful response in contexts of fragile human ties]. EPISTEME KOINONIA, 3(5), 27-50. http://dx.doi.org/10.35381/e.k.v3i5.527

Rodríguez, D., Erazo, J., \& Narváez, C. (2019). Técnicas cuantitativas de investigación de mercados aplicadas al consumo de carne en la generación millennial de la ciudad de Cuenca (Ecuador) [Quantitative market research techniques] Recuperado de: https://url2.cl/ibtfw. Revista Espacios, 20.

Salgado, J. A., Leria, F. J., Pilar Franco, M. E., \& Gajardo, X. R. (2017). Efecto de la Motivación sobre la Profundidad en los Procesos de Estudio en Universitarios de Formación en Pedagogía [Effect of Motivation on the In-depth Study Processes Among]. REICE. Revista Iberoamericana sobre Calidad, Eficacia y Cambio en Educación, 87-105. doi:http://dx.doi.org/10.15366/reice2017.15.4.005

Tapia-Vélez, J., García-Herrera, D., Erazo-Álvarez, J., \& Narváez-Zurita, C. (2020). Aprendizaje Basado en Problemas como estrategia didáctica para el desarrollo del razonamiento lógico matemático. Revista Arbitrada Interdisciplinaria Koinonía, 5(1), 753-772. http://dx.doi.org/10.35381/r.k.v5i1.808

Ventura, T. L. (2018). La educación física y las relaciones sociales en educación primaria. [Physical education and social relationship in primary education]. Revista Infad de Psicología, 1-18. Obtenido de https://n9.cl/apqh 
EPISTEME KOINONIA

Revista Electrónica de Ciencias de la Educación, Humanidades, Artes y Bellas Artes

Año III. Vol III. N 6 . Julio - Diciembre 2020

Hecho el depósito de Ley: FA2018000022

ISSN: 2665-0282

FUNDACIÓN KOINONIA (F.K)

Santa Ana de Coro, Venezuela

Damián Fernando Montalván-Larco; Darwin Gabriel García-Herrera; Carlos Marcelo Ávila-Mediavilla; Juan Carlos Erazo-Álvarez

Zapatero, A. (2017). Beneficios de los estilos de enseñanza y las metodologías centradas en el alumno en educación física [Benefits of teaching styles and student-centred methodologies in Physical Education]. E-balonmano.com: Revista de Ciencias del Deportem, 237-250. Obtenido de https://n9.cl/xyk76

C2020 por el autor. Este artículo es de acceso abierto y distribuido según los términos y condiciones de la licencia Creative Commons Atribución-NoComercial-Compartirlgual 4.0 Internacional (CC BY-NC-SA 4.0) (https://creativecommons.org/licenses/by-nc-sa/4.0/). 